\title{
A gene catalogue for post-diapause development of an anhydrobiotic arthropod Artemia franciscana Wie-Hua Chen ${ }^{\dagger 1,2}$, Xiaomeng Ge ${ }^{\dagger 1}$, Weiwei Wang1, Jun $\mathrm{Yu}^{* 1}$ and Songnian $\mathrm{Hu}^{* 1}$
}

Address: ${ }^{1}$ CAS Key Laboratory of Genome Sciences and Information, Beijing Institute of Genomics, Chinese Academy of Sciences, Beijing, 100029, PR China and 2European Molecular Biology Laboratory (EMBL), Meyerhofstrasse 1, 69117 Heidelberg, Germany

Email: Wie-Hua Chen - chenwh550@gmail.com; Xiaomeng Ge - gexiaomeng@big.ac.cn; Weiwei Wang - wangweiwei@big.ac.cn; Jun Yu* - junyu@big.ac.cn; Songnian Hu* - husn@genomics.org.cn

* Corresponding authors †Equal contributors

Published: 27 January 2009

BMC Genomics 2009, 10:52 doi:10.1186/147|-2/64-10-52
Received: 28 October 2008

Accepted: 27 January 2009

This article is available from: http://www.biomedcentral.com/I47I-2/64/I0/52

(C) 2009 Chen et al; licensee BioMed Central Ltd.

This is an Open Access article distributed under the terms of the Creative Commons Attribution License (http://creativecommons.org/licenses/by/2.0), which permits unrestricted use, distribution, and reproduction in any medium, provided the original work is properly cited.

\begin{abstract}
Background: Diapause is a reversible state of developmental suspension and found among diverse taxa, from plants to animals, including marsupials and some other mammals. Although previous work has accumulated ample data, the molecular mechanism underlying diapause and reactivation from it remain elusive.

Results: Using Artemia franciscana, a model organism to study the development of post-diapause embryos in Arthropod, we sequenced random clones up to a total of 28,039 ESTs from four cDNA libraries made from dehydrated cysts and three time points after rehydration/reactivation, which were assembled into 8,018 unigene clusters. We identified 324 differentially-expressed genes (DEGs, $P<0.05$ ) based on pairwise comparisons of the four cDNA libraries. We identified a group of genes that are involved in an anti-water-deficit system, including proteases, protease inhibitors, heat shock proteins, and several novel members of the late embryogenesis abundant (LEA) protein family. In addition, we classified most of the up-regulated genes after cyst reactivation into metabolism, biosynthesis, transcription, and translation, and this result is consistent with the rapid development of the embryo. Some of the specific expressions of DEGs were confirmed experimentally based on quantitative real-time PCR.
\end{abstract}

Conclusion: We found that the first 5-hour period after rehydration is most important for embryonic reactivation of Artemia. As the total number of expressed genes increases significantly, the majority of DEGs were also identified in this period, including a group of water-deficientinduced genes. A group of genes with similar functions have been described in plant seeds; for instance, one of the novel LEA members shares $~ 70 \%$ amino-acid identity with an Arabidopsis EM (embryonic abundant) protein, the closest animal relative to plant LEA families identified thus far. Our findings also suggested that not only nutrition, but also mRNAs are produced and stored during cyst formation to support rapid development after reactivation. 


\section{Background}

Diapause, also known as discontinuous development, is a reversible state of developmental suspension often promoted by seasonal environmental adversity cues. It is a strategy that ensures successful species survival through timing the post-diapause development for favorable environmental conditions. This protective mechanism is widely distributed among different taxa, including plants, insects, vertebrates, and even some mammals and it occurs at different developmental stages, such as embryonic, larval, pupal, or adult stages, often varying from species to species.

Artemia is a group of small ancient crustaceans living in saline waters. Induced by signals from forthcoming seasonal adversities (not by the adversities themselves), Artemia produces shelled embryos (cysts) that suspend development and metabolism at the gastrula stage, which contain around 4,000 cells [1,2]. These cysts are in a state of diapause, a physiological dormancy with specific releasing conditions. The primary important feature of diapause is that once initiated, it can only be released by certain stimuli; until then, the cysts wouldn't continue to develop even putting them into favorable environments. This feature is essential in distinguishing diapause as a different phenomenon from other forms of dormancy such as hibernation and quiescence [3]. In Artemia, the diapause could be broken by dehydration (often complete dehydration is required) and is followed by a state of quiescence, from which the cysts can begin direct development, should conditions change to become more favorable. The diapause-broken was often considered as a mechanical process instead of physiological transition, because a) the time of the dehydration process doesn't matter to the hatching rate; b) multiple-round of dehydration- rehydration is sometime necessary to diapausebreak and c) little physiological and biological changes were observed [4]. These dehydrated cysts are able to survive years even decades, with little signs of metabolism and energy consumption, but remain viable [5-7]. Once reactivated in favorable environments, they resume development and give rise to free-swimming larva within 24 hours. The developing of embryonic development of Artemia is generally divided into three stages, namely prediapause, diapause and post-diapause [4].
Physiologically and biologically, the encysted embryos resemble plant seeds in many aspects. For example, both contain embryos that develop to certain stages and undergo complete dehydration without any obvious loss of viability even stored for years. Recent studies also revealed that certain molecules such as heat shock proteins are significantly enriched in both cysts and plant seeds.

As an initial step to address the molecular mechanisms of diapause, we first of all constructed a cDNA library using dehydrated cysts that were in a state of quiescence and likely to contain genes important to the maintenance of diapause, since no physiological changes observed during the transition. Then we analyzed its gene expression profiles and compared with that of cDNA libraries that were derived from three time points of subsequent development after rehydration. Expression sequence tags (ESTs) were used to get quick access to gene sequences and expression information of the developing Artemia. We obtained 30,000 high-quality ESTs from the four cDNA libraries in approximately equal numbers from each, and clustered them into 8,018 unigenes after sequence assembly and annotation. We also validated a few gene expression profiles using quantitative real-time PCR. All EST sequences were deposited into NCBI's dbEST under accessions from ES492186 to ES529129.

\section{Results and discussions}

\section{Library construction and sequence assembly}

We constructed four directional, non-normalized cDNA libraries for the brine shrimp, Artemia franciscana, using RNA samples extracted from dehydrated cysts (post-diapaused cysts) as well as three developmental stages at 5 , 10,15 hours after rehydration and named them as AfD0, AfR5, AfR10 and AfR15, respectively. We randomly sequenced $\sim 10,000$ clones from the 5 '-end. After eliminating host gene contaminations, trimming vector and lowquality ( $<100 \mathrm{bp}$ in length) sequences, we obtained 28,039 high-quality EST sequences with an average length of 471 bp. The sequences were clustered into 8,018 unigenes that contain 1,951 contigs and 6,067 singlets (Table $1)$. The largest cluster has 601 ESTs.

Table I: Summary of cDNA libraries

\begin{tabular}{ccccc}
\hline Library & Clone & Acquired Sequence & High-Quality Sequence & Assembled Unigene \\
\hline AfD0 & 9,002 & 8,130 & 7,567 & 1,698 \\
AfR5 & 8,178 & 7,453 & 6,832 & 3,146 \\
AfRI0 & 8,046 & 7,543 & 6,901 & 2,787 \\
AfRI5 & 7,988 & 7,473 & 6,739 & 3,006 \\
Total & 33,214 & 30,599 & 28,039 & 8,018 \\
\end{tabular}




\section{Annotation of Artemia unigenes}

We annotated the Artemia unigenes by comparing to protein sequences collected from public databases (Figure 1), and 3,953 unigenes (49.3\%) showed significant sequence similarities (e-value $<1 e-5)$ to known genes. To determine whether the low sequence similarities of the remaining unigenes were attributable to short open reading frames (ORFs), we used getorf [8] to examine their putative ORFs. The results indicated that only $2.6 \%$ of the non-matching unigenes contained ORFs less than 30 amino acids. We noted that an even smaller portion $(3,214)$ of the unigenes had shown significant similarities with the prerelease genome annotation FM3 (filtered models release 3) of Daphnia, a close relative of Artemia that shared many physiological similarities, indicating high genetic diversities among Crustacean. An overview of functional categories (Gene Ontology, GO) of annotated unigenes is shown in Figure 2.

\section{Expression profiles of Artemia unigenes in four cDNA libraries}

We obtained 1,698 unigenes from AfD0 that derived from dehydrated cysts where metabolism and development are nearly suspended. We obtained significantly more unigenes from the other three cDNA libraries: 3,146, 2,787, and 3,006 from AfR5, AfR10, and AfR15, respectively (Table 1). We also carried out pair-wise comparisons for expression profiles among the libraries. For example, we identified 362 unigenes expressed in AfD0 and AfR5, 560 in AfR5 and AfR10, and 558 in AfR10 and AfR15. Only 194 unigenes were found universally expressed and nearly
$50 \%$ unigenes were found unique to each library. Expression abundances of the unigenes correlate to their distributions in four libraries. For example, universally presented genes were highly expressed, with a mean cluster size of 46.7; the cluster sizes of those unique unigenes strongly biased toward very low values (around one EST per unigene). Genes expressed in two libraries showed inbetween abundances with a mean cluster size of 21.2.

To identify differentially expressed genes (DEGs) that are statistically significant, we adopted the general Chi-square algorithm to test expression profiles as previous studies suggested it was applicable and reliable for EST analysis [9]. We identified 324 DEGs with p-value $<0.05$, including 279 between AfD0 and AfR5, 64 between AfR5 and AfR10, and 50 between AfR10 and AfR15. This algorithm masked most of the unigenes that were unique to any of the cDNA libraries as only 21 of them were assigned as DEGs. Our results suggested that a major change occurred in the first five hours after reactivation. We annotated 56 up-regulated and 77 down-regulated genes from 133 DEGs between AfD0 and AfR5 (47.3\%, out of 279 DEGs). We assigned one or more GO terms to all these 133 genes by an annotation transfer process (see Materials and Methods). As shown in Figure 3, GO categories of cell, organelle, protein complex, structural molecular activity, cellular process, and physiological process are most abundant among down-regulated DEGs, and protein complex, enzyme regulator activity, and response to stimulus are abundant among up-regulated DEGs. A full list of DEGs is shown in Additional File 1.

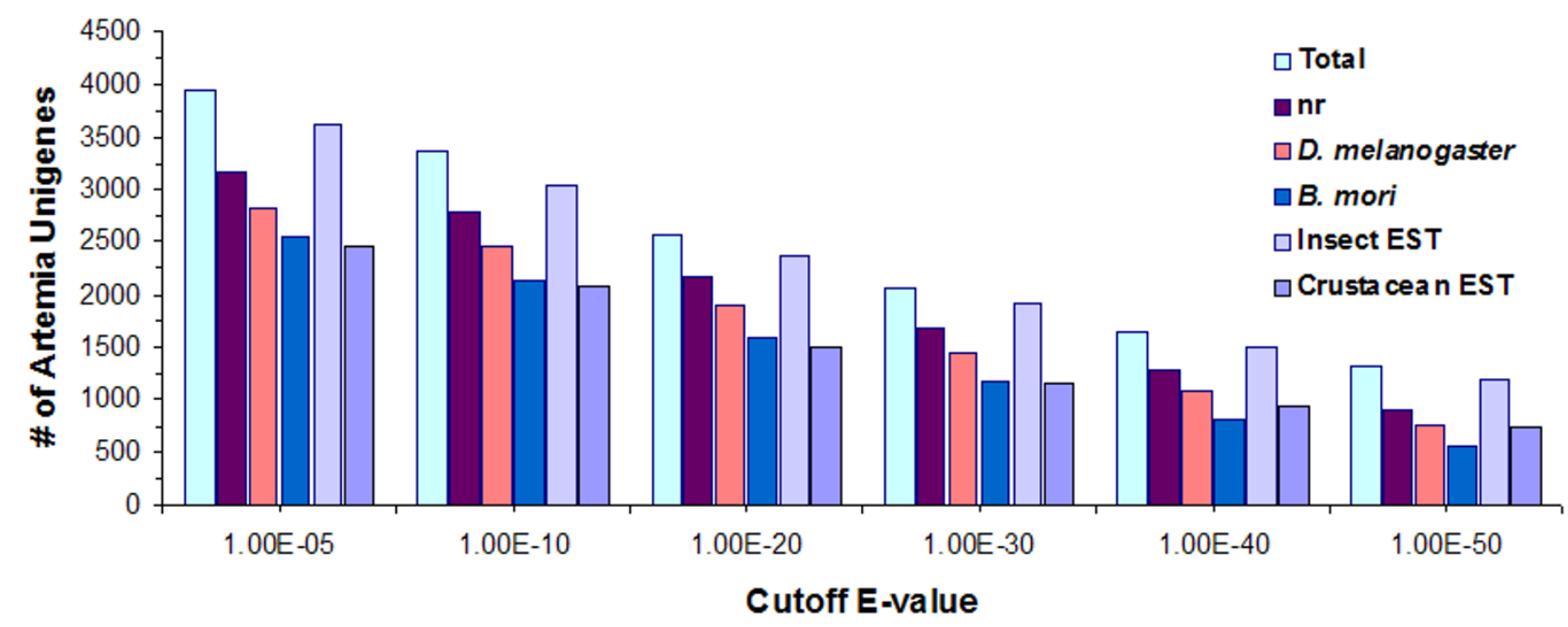

Figure I

Sequence comparisons with other relevant species. Numbers of Artemia unigenes significantly matched to sequences in NCBI's non-redundant protein ( $\mathrm{nr}$ ) database, Drosophila melanogaster protein database (release 4.3), silkworm (Bombyx mori) BGF protein database, and other available EST sequences of Insecta and Crustacea from dbEST. 

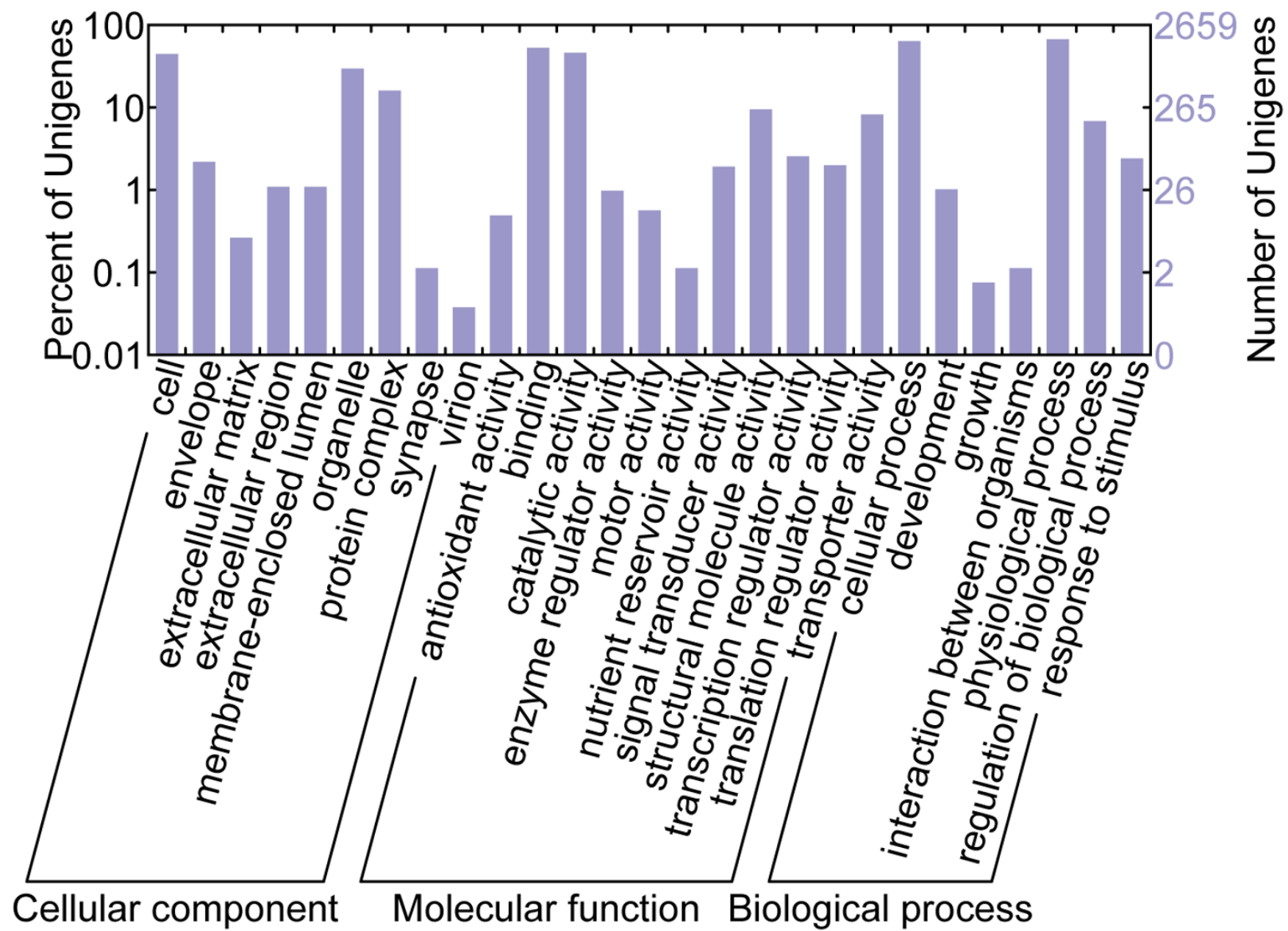

Figure 2

Functional categorization of Artemia unigenes. Gene Ontology classifications of Artemia unigenes with significant hits in Uniprot.

Down-regulated and up-regulated DEGs after reactivation We identified 142 down-regulated genes after reactivation and annotated 77 of them. We grouped cysts-enriched transcripts into several categories based on their putative biological functions, such as stored in cysts and utilized after reactivation or involved in anti-desiccation and diapause. We also look into ribosomal proteins as they are stored in cysts and used after reactivation to facilitate quick physiological and biological transition from the quiescence to the active states [10], and identified 37 ribosomal protein coding genes as down-regulated DEGs. In addition, we noticed a cathepsin-like cysteine proteinase (CLAP) that is stored in cysts but activated after rehydration and involved in yolk utilization in Artemia embryos [11]. However, other studies shown that proteinases were involved in desiccation-tolerance and the accumulation of proteases was often correlated with increased amount of aggregated/denatured proteins induced by water-loss [12]. As proteases are often multi-functional, our efforts to assign them into function-related groups became difficult sometimes. For example, we identified another down-regulated HAD-like protease (haloacid dehalogenase-like hydrolase) but categorized it as waterloss induced genes.

Water-deficit induces the expression of protease inhibitors and chaperones and serve as counteracting mechanisms to prevent protein degradation. Among down-regulated DEGs, we identified a cysteine protease inhibitor (Cystatin B) that binds tightly to and inhibit papain, cathepsin $B$ and lysosomal cathepsin L [13] and several chaperone genes, including late embryogenesis abundant proteins (LEAs), a small heat shock protein p26, and HSP70. As classic chaperone, p26 was previous found abundantly 


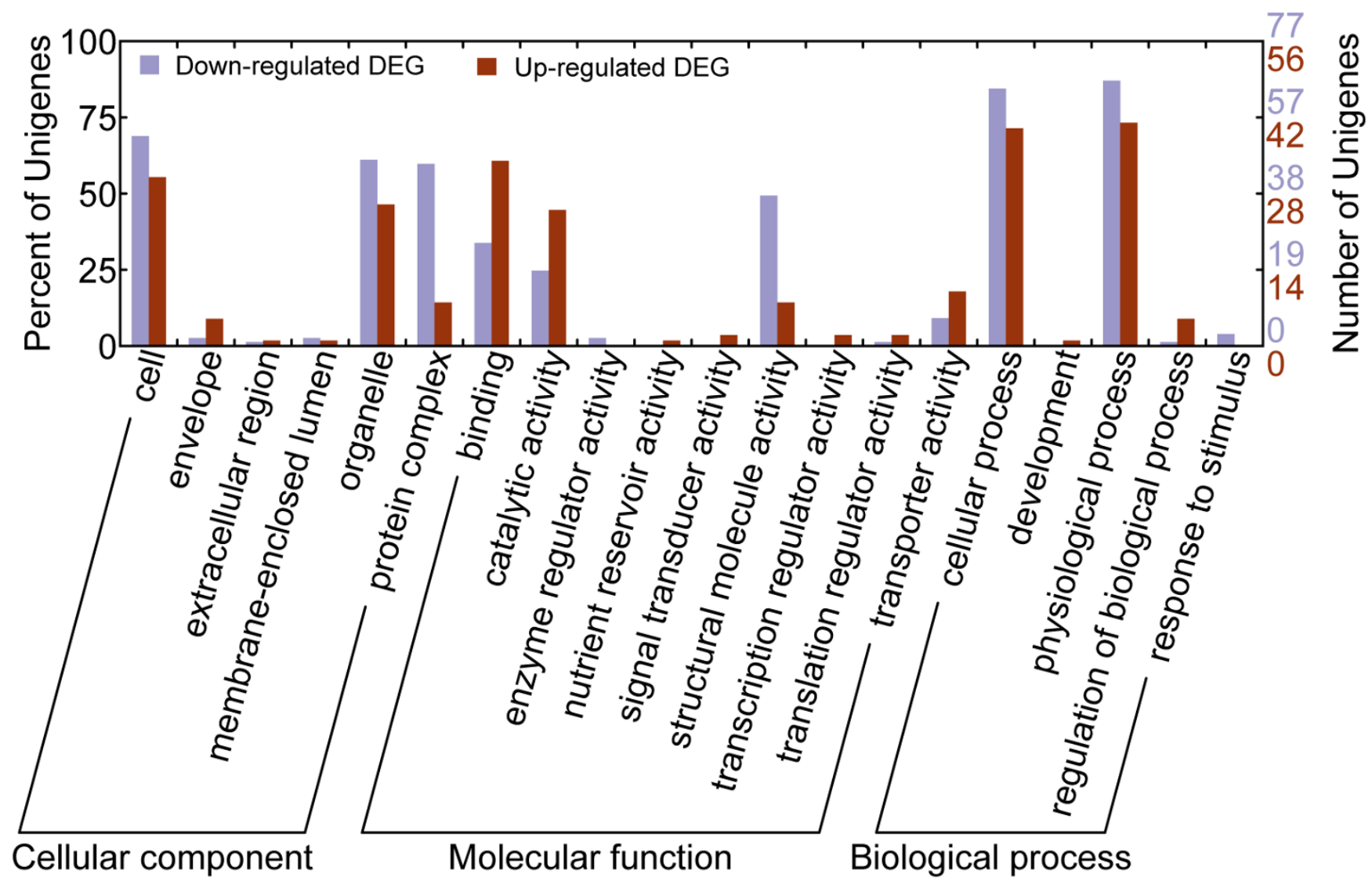

Figure 3

GO classification of differentially-expressed genes (DEGs) between diapause cysts (AfD0) and developing Artemia in early developmental stage (AfR5).

expressed in encysted embryos of Artemia and it was suggested to protect proteins from irreversible denaturation in an energy-independent manner [14] and cells against oxidative damage [15]. In stressed cells, HSP70 and p26 move to the nucleus upon stresses and played a role in stabilization of nuclear matrix proteins [16].

LEAs are a group of new and non-classic chaperones. They were originally identified in plant seeds and recently in animals. Considerable evidence suggested that LEA proteins were involved in desiccation resistance, by means of water retention, sequestration of ions, direct protection of other proteins or membranes $[12,17]$, although the exact molecular mechanisms how these protein function in the cell remain elusive. From the 8,018 unigenes, we identified three LEA genes, including two novel members, Afr_EM-like and Afr_LEA3, and both were down-regulated after reactivation. As shown in Figure $4 \mathrm{a}$, their expression patterns were consistent with their putative functions in desiccation tolerance. Both encode strongly hydrophilic proteins (Figure 4b). The novel Afr_EM-like protein contains a LEA_5 domain (PF00477) with an e- value of $8.6 e-07$ in PFAM database, and showed $65 \%$ amino acid identity and $74 \%$ similarity to the Em (embryonic abundant, GenBank accession NP 190749, Figure 4c) protein of Arabidopsis thaliana, which is the closest LEA relative in plants identified so far. The other novel Afr_LEA3 gene coded for a protein containing a LEA_3 domain, and had best match with LEA3 protein (GenBank Accession XP 001675059) of Caenorhabditis briggsae with relatively low similarity (27\% identities and 45\% similarity, Figure 4d). Two LEA genes, AfrLEA1 and AfrLEA2, were reported previously and showed to have a differential expression between post-diapaused embryos and freeswing larva of Artemia franciscana based on quantitative real-time PCR (qRT-PCR) [18]. However, only AfrLEA2 was identified in our cDNA library, which matched to a single EST and did not show differential expression among our cDNA libraries. The discrepancy was most likely attributable to different technique routes adopted in the two studies: qRT-PCR is much more sensitive to low abundant genes, while cDNA cloning can only capture abundantly expressed transcripts. We also identified many other genes that are significantly enriched in dehy- 
a

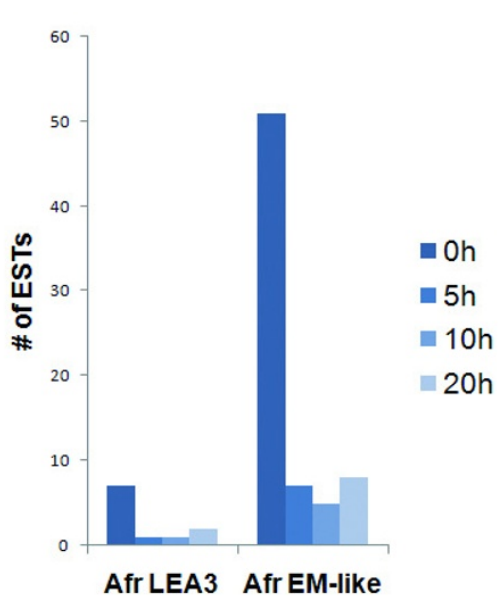

b

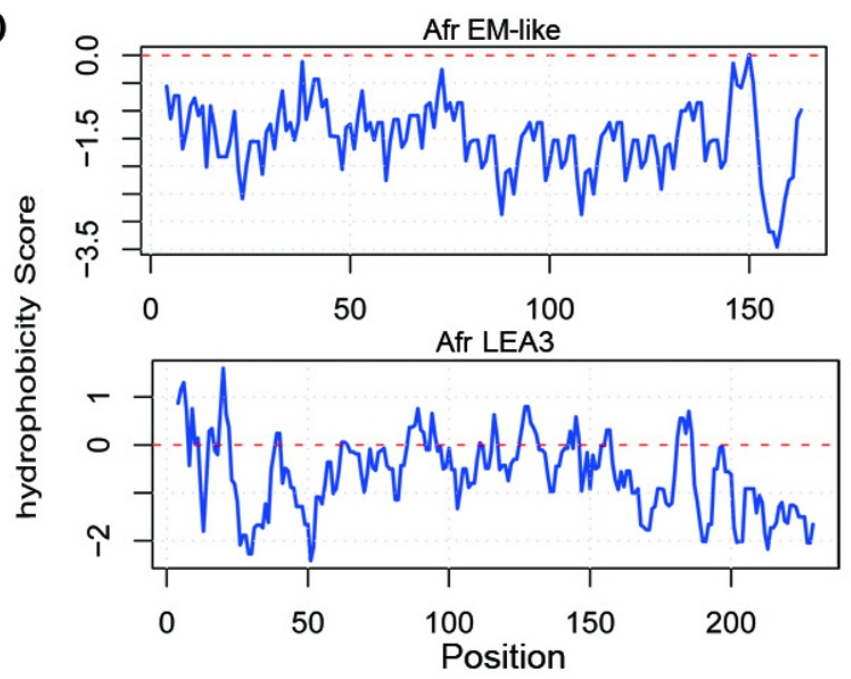

C

Ath_ATEM1/1-152 Afr_EM-like/1-166

Ath_ATEM1/1-152 Afr_EM-like/1-166

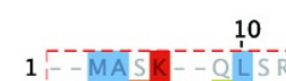

10

$20 \quad 30 \quad 40$

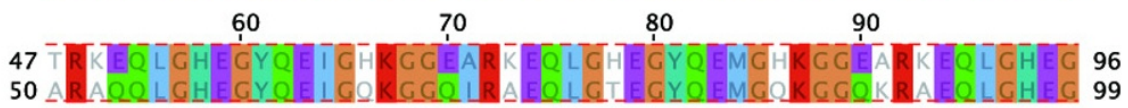

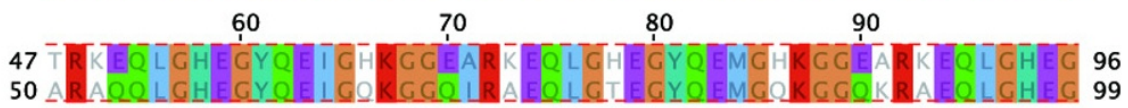

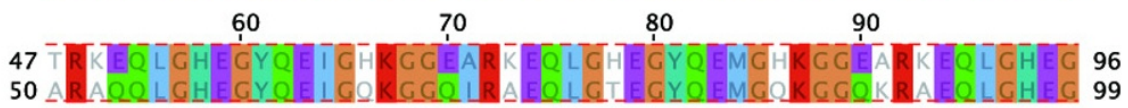

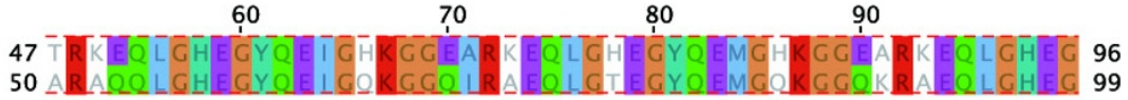

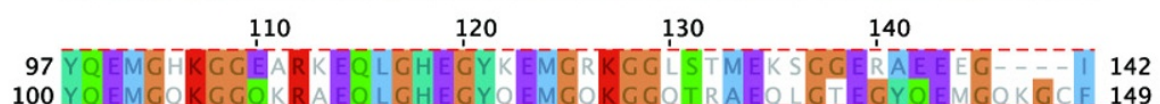

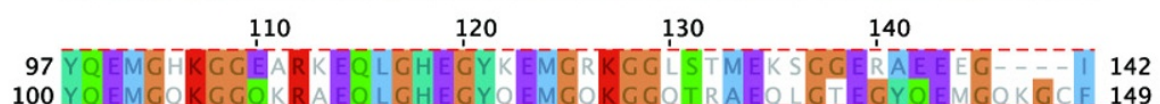

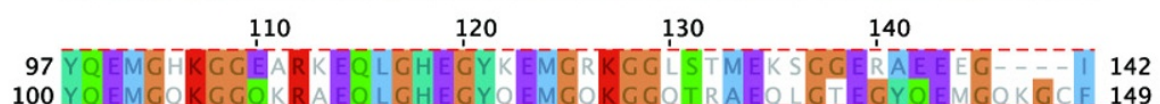

Ath_ATEM1/1-152

Afr_EM-like/1-166

Ath_ATEM1/1-152

160

Afr_EM-like/1-166 150 S_SP_P_K K K K N N E E__CP E $\perp$ C

d

Afr_LEA_group3/1-232

Cbr_LEA_group3/1-324

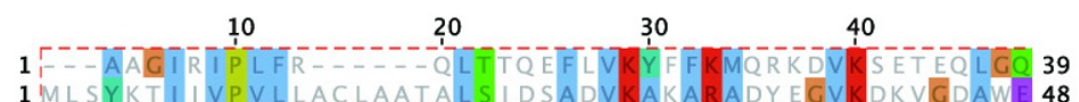

Afr_LEA_group3/1-232 Cbr_LEA_group3/1-324

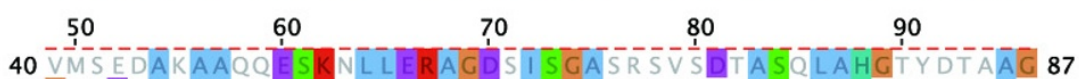

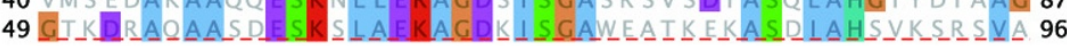

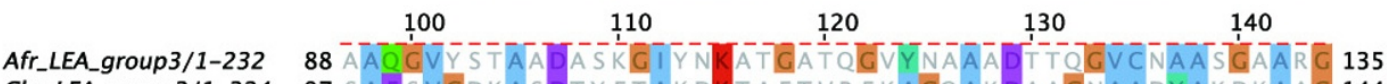
Cbr_LEA_group3/1-324 97 SAESVGDKASDT_KEIAKDKIAEIVR_E_AGQAKDAAGNAADYAKDKAAG 144

$$
150
$$

160

170

180

190

Afr_LEA_group3/1-232 136 A Y D TA S G L TOATLD

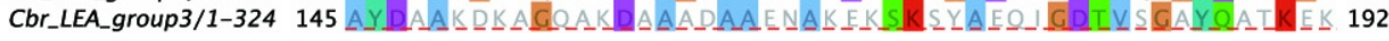

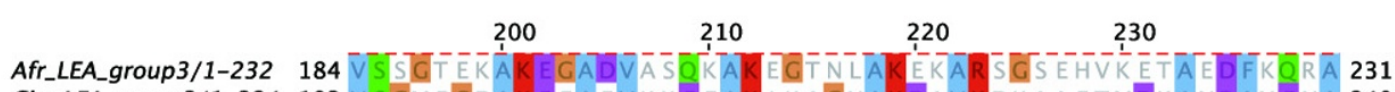
Cbr_LEA_group3/1-324 193 VS_CMEGDAKEEAEVIKEEAKLIAGNAKDAVIVDKA_EMDKANDAYENA 240 Afr_LEA_group3/1-232 $232 \mathrm{~S}$

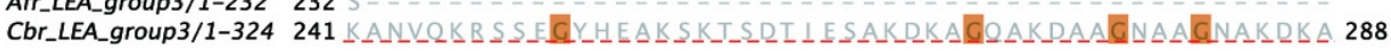
Afr_LEA_group3/1-232 3003003

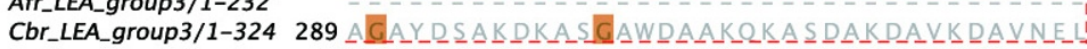

Figure 4

Expression abundances and sequence features of two novel LEA genes in Artemia. 
drated embryos, including SEC-like, elongation factor 2 (EF-2), larval cuticle and chitin binding proteins.

In a previous study, Qiu Z. et al identified $\sim 85$ genes enriched in the diapause-destined embryos of Artemia franciscana by using a subtractive cDNA library [19]. We found only nine down-regulated DEGs that are shred between the two datasets, including those of p26, superoxide dismutase, and several ribosomal proteins (Additional file 2). Since only less than 300 cDNA clones were sequenced in the study and our sampling depth is also rather shallow, we did not anticipate much overlapping data.

Through our annotation pipeline, we were able to annotate 56 of 137 up-regulated DEGs in four CDNA libraries. From them, we identified several functionally related groups, including those involved in (1) metabolism and ATP generation, (2) cell growth and cell division, (3) transcription and translation, and (4) folding of newly translated proteins. The increasing expression of these genes was consistent with the rapid development after reactivation of Artemia cysts, which usually gives rise to free-swimming larva within 24 hours.

\section{Validation of EST expressions by qRT-PCR}

To validate our EST sequencing results, we performed quantitative RT-PCR) for nine selected genes that showed differential expression, in which six down-regulated and three up-regulated DEGs. The down-regulated genes are three stress-tolerance genes, p26, HSP70 and LEA, two proteases, CLAP and HAD-like, and a protease inhibitor, Cystatin B inhibitor-like; three up-regulated genes are cellcycle regulator CDC48, chaperonin-containing TCP that help fold newly translated proteins, and a UCP2. Since all these DEGs showed differential expression between $0 \mathrm{~h}$ and $5 \mathrm{~h}$, we only performed qRT-PCR experiments using mRNA that were used to construct these two cDNA libraries. The results confirmed the expressions (Table 2).

\section{Conclusion}

In this report, we described 28,039 Artemia franciscana ESTs and their 8,018 unigenes, representing the largest sequence resource in the public databases for this organism. We confirmed differential expression for p26, a small heat shock protein that was abundantly expressed in encysted embryos and that serves as a multifunctional molecular chaperone. Comparing to a public available dataset that derived from diapause-destined Artemia embryos, we noticed the critical role of p 26 in both entering and maintaining dipause. We also identified two novel late embryo abundant (LEA) genes homologous to the plant LEA proteins, and together with HSP70, proteases, and protease inhibitors, these down-regulated genes after cysts reactivation provided the ability of desic-
Table 2: qRT-PCR validation of selected DEGs

\begin{tabular}{lrr}
\hline Gene & \multicolumn{2}{c}{ Expression Change* } \\
& ESTs & Q-RT-PCR \\
\hline HSP26 & -3.8 & -5.08 \\
Cystatin B inhibitor & -7 & -2.06 \\
LEA & -7.3 & -7.10 \\
cathepsin L-like protease & -3.4 & -5.29 \\
haloacid dehalogenase-like & -1.5 & -3.55 \\
HSP70 & -11 & -3.57 \\
CDC48 & $0 / 5$ & +3.38 \\
chaperonin-containing TCP & +3.33 & +3.66 \\
UCP2 & +2.5 & +10.55 \\
GADPH & & \\
\hline
\end{tabular}

* Fold changes are used for expression quantization; plus and minus indicate down- and up-regulations. For the EST data, we used transcripts per million (TPM) instead of absolute expression abundance, and for Q_PCR results, we calculated fold changes directly, according to the manufacture's instruction.

cation tolerance for the encysted embryos. A similar set of genes have also been reported in plant seeds [12].

Among down-regulated DEGs, we identified 37 ribosomal protein coding genes; this is consistent with a previous finding that mRNA activity for ribosomal proteins is stored in the cytoplasm of dormant cysts and associated with polysomes [10]. Other protein coding genes may also be stored in encysted embryos. For example, a cathepsin like cysteine proteinase that was involved in yolk utilization in Artemia embryos [11] was enriched in dehydrated embryos. Several groups of functionally related genes were up-regulated up to 20 folds after reactivation, which include genes that are involved in energy generation, translation/transcription, metabolism, growth, cell division, and differentiation. The activation of these genes allows Artemia embryos to convert the stored yolk palette into energy and the result is consistent with their rapid development after reactivation.

\section{Methods \\ Sample preparation}

We obtained dehydrated Artemia franciscana cysts that were in the quiescence state from Dr. Jianhai Xiang's laboratory at the Institute of Oceanology, Chinese Academy of Sciences, Qingdao, China. We confirmed the taxonomic identity based on mitochondrial $\operatorname{cox} 1$ sequence from single cysts, a technique known as DNA Bar-coding. We evaluated the hatching rate of the cysts following a previously-described procedure [20] to optimize hatching rates. The first free-swing larva usually appear at the $10^{\text {th }}$ hour of hatching, $15 \%$ of the cysts hatch after 15 hours, and more than $90 \%$ of the cysts develop into free-swing larva at the $20^{\text {th }}$ hour. We started our experiments from dehydrated cysts and collected samples from the rehy- 
drated cysts after 5-, 10-, and 15-hour hatching for the cDNA library construction.

\section{cDNA library construction and quality estimation}

We extracted total RNAs from the samples using Trizol agent (Invitrogen), and isolated polyA mRNAs using PolyATtract mRNA isolation system (Promega). To obtain a broad coverage of Artemia transcripts, we size-fractioned double-stranded cDNAs before cloning. We constructed cDNA libraries using the directional pBluescript ${ }^{\circledR}$ II XR vector (Stratagene), exploiting the EcoRI and XhoI restriction sites, according to the manufacturer's instruction. The cDNA libraries were not normalized. To assess the quality of cDNA libraries, we performed colony PCR on 96 randomly picked clones to determine the average insert size and percentage of clones without inserts and sequenced 384 randomly picked clones from each cDNA library to determine the ratio of host and vector sequences (empty clones), the sequence length after masking vector sequences, and the ratio of unique sequences (contigs + singlets)/reads.

\section{EST sequencing, assembly, and annotation}

We acquired 5' ESTs from $~ 10,000$ colonies of each library, using MegaBase ${ }^{\circledast} 1000$ sequencers. We assembled the sequences using Phred-phrap-consed [21,22] with default parameters after removal of vector and low-quality (< 100 bp) sequences. We annotated our unigene sequences (consensus sequences of assembled clusters, including contigs and singlets) based on protein sequences in NCBI non-redundant (nr) protein database and several other public databases including Drosophila melanogaster protein database (dmel-all-translation; release 4.3) from FlyBase [23] and silkworm Bombyx mori BGF protein database database from SilkDB [24], using BLAST [25] based tools, such as blastx and tblastx. We also collected available EST sequences of Insecta and Crustacean from NCBI's dbEST. To classify the Artemia unigenes into Gene Ontology (GO) categories, we compared them with proteins in UniProt (uniprot_sprot and uniprot_trembl) and assigned GO terms according to their best matches, by using a UniProt2GO data provided by the European Bioinformatics Institute (EBI).

\section{Identification of differentially-expressed genes (DEGs) and quantitative $P C R$ validation}

We used a program IDEG6 $[9,26]$ to identify genes that are differentially expressed among libraries. A unigene (or gene) is said to be differentially expressed when it produces a $P<0.05$ using Chi-Square algorithm. We further validate the expression profiles of nine DEGs, using quantitative real-time PCR (qRT-PCR). We designed primers for these genes, using a program OLIGO6 with the following parameters: $\mathrm{Tm}, 60 \pm 2{ }^{\circ} \mathrm{C}$, difference $<=2$ between a pair of primers; primer length, 17-22 bp; GC content, 40-
60\%; PCR product length, 150-200 bp. Whenever possible, we chose primers that locate at the 3 ' end of transcripts. A full list of designed primers is shown in Table 2 and their sequences in Additional file 3.

We used the same RNA samples as what for constructing the cDNA libraries. The RNA samples were treated with RNase-free DNase I (Promega) to remove possible DNA contaminations. The first-strand cDNA was synthesized by using 500 ng total RNAs, poly(T) primers and SSII reverse polymerase (Invitrogen). qRT-PCR was conducted by using a Quant SYBR Green PCR kit (Tiangen, China). We chose the GADPH gene as an external reference for data normalization. The PCR reaction parameters were as follows: $95^{\circ} \mathrm{C}$ for $2 \mathrm{~min}$; 40 cycles of 3-temperature of $95^{\circ} \mathrm{C}$ for $15 \mathrm{~s}, 60^{\circ} \mathrm{C}$ for $20 \mathrm{~s}, 72^{\circ} \mathrm{C}$ for $30 \mathrm{~s}$. Three replicates for each pair of primers per template were included. qRT-PCR data were analyzed by using Opticon Monitor ${ }^{\circledR}$ software. Melting curves for each PCR were carefully analyzed to avoid non-specific amplifications. Gene expressions were quantified and transformed by using the $\Delta \mathrm{Ct}$ formula normalized with the expression of GAPDH.

\section{Authors' contributions}

WHC, XG and WW carried out the experiments and data analysis; WHC wrote the manuscript; JY and SH designed and supervised this research and revised the manuscript.

\section{Additional material}

\section{Additional file 1}

Differentailly expressed genes (DEGs) between cDNA libraries AfDo and AfR5. The data provided a complete list of differentially expressed genes we identified between $A f D 0$ and AfR5.

Click here for file

[http://www.biomedcentral.com/content/supplementary/1471-

2164-10-52-S1.xls]

\section{Additional file 2}

down-regulated DEGs shared between this study and published data. We compared the down-regulated DEGs that were mostly enriched in dehydrated cysts in this study with genes enriced in diapause-destined Artemia embryos from a previous study (see Ref 19) and provided the gene list here.

Click here for file

[http://www.biomedcentral.com/content/supplementary/14712164-10-52-S2.xls]

\section{Additional file 3}

primer sequences used in qRT-PCR experiments. This file provided primer sequences that we designed for $q R T-P C R$ experiments. Click here for file

[http://www.biomedcentral.com/content/supplementary/14712164-10-52-S3.xls] 


\section{Acknowledgements}

We would like to thank Dr. Jianhai Xiang of Institute of Oceanology, Chinese Academy of Sciences, for kindly providing Artemia cysts. The work is supported by grants from Chinese Academy of Sciences (KSCX2-SW-223 to Jun Yu), Chinese Natural Science Foundation (30270748 to Jun Yu), and Ministry of Sciences and Technology (2005AA235II0 to Jun Yu).

\section{References}

I. Anderson E, Lochhead JH, Lochhead MS, Huebner E: The origin and structure of the tertiary envelope in thick-shelled eggs of the brine shrimp, Artemia. J Ultrastr Res 1970, 32(5-6):497-525.

2. Morris JE, Afzelius BA: The structure of the shell and outer membranes in encysted Artemianext term salina embryos during cryptobiosis and development. J Ultrastr Res 1967, 20(34):244-259.

3. Taylor F: Insect Life Histories: Seasonal Adaptations of Insects. Science 1986, 232(4754): II52.

4. Abatzopoulos TJB, J A, Clegg JS, Sorgeloos P: 2002 [http:// www.springer.com/life+sci/zoology/book/978-I-4020-0746-0]. Artemia: Basic and Applied Biology: Springer

5. Warner $\mathrm{AH}$, Clegg JS: Diguanosine nucleotide metabolism and the survival of artemia embryos during years of continuous anoxia. Eur J Biochem 200I, 268(6): I568-I576.

6. Clegg J: Embryos of Artemia franciscana survive four years of continuous anoxia: the case for complete metabolic rate depression. J Exp Biol 1997, 200(Pt 3):467-475.

7. Hontoria F, Crowe JH, Crowe LM, Amat F: METABOLIC HEAT PRODUCTION BY ARTEMIA EMBRYOS UNDER ANOXIC CONDITIONS. J Exp Biol 1993, I78(I I49-I59 [http:// jeb.biologists.org/cgi/content/abstract/178////49].

8. Rice P, Longden I, Bleasby A: EMBOSS: the European Molecular Biology Open Software Suite. Trends Genet 2000, 16(6):276-277.

9. Romualdi C, Bortoluzzi S, Danieli GA: Detecting differentially expressed genes in multiple tag sampling experiments: comparative evaluation of statistical tests. Hum Mol Genet 200I, 10(19):2|33-2|4|.

10. Pierandrei-Amaldi P, Campioni N: Messenger RNA for ribosomal proteins in dormant and developing Artemia salina embryos. Biochim Biophys Acta 198I, 655(3):359-365.

11. Warner AH, Perz MJ, Osahan JK, Zielinski BS: Potential role in development of the major cysteine protease in larvae of the brine shrimp Artemia franciscana. Cell Tissue Res 1995, 282(I):2I-3I.

12. Bray EA: Molecular Responses to Water Deficit. Plant Physiol 1993, 103(4): 1035-1040.

13. Abrahamson M, Alvarez-Fernandez M, Nathanson CM: Cystatins. Biochem Soc Symp 2003:179-199.

14. Day RM, Gupta JS, MacRae TH: A small heat shock/alpha-crystallin protein from encysted Artemia embryos suppresses tubulin denaturation. Cell Stress Chaperones 2003, 8(2): 183-193.

15. Collins $\mathrm{CH}$, Clegg JS: A small heat-shock protein, p26, from the crustacean Artemia protects mammalian cells (Cos-I) against oxidative damage. Cell Biol Int 2004, 28(6):449-455.

16. Willsie JK, Clegg JS: Small heat shock protein p26 associates with nuclear lamins and HSP70 in nuclei and nuclear matrix fractions from stressed cells. J Cell Biochem 2002, 84(3):60I-6I4.

17. Ingram J, Bartels D: The Molecular Basis Of Dehydration Tolerance In Plants. Annu Rev Plant Physiol Plant Mol Biol 1996, 47:377-403.

18. Hand Steven C, J D, M M A, W T L: Life without water: expression of plant LEA genes by an anhydrobiotic arthropod. Journal of Experimental Zoology Part A: Ecological Genetics and Physiology 2007, 307A(I):62-66.

19. Qiu Z, Tsoi SC, MacRae TH: Gene expression in diapause-destined embryos of the crustacean, Artemia franciscana. Mech Dev 2007, I 24(I I- | 2):856-867.

20. Wang W, Meng B, Chen W, Ge X, Liu S, Yu J: A proteomic study on postdiapaused embryonic development of brine shrimp (Artemia franciscana). Proteomics 2007, 7(1 9):3580-3591.

21. Ewing B, Green P: Base-Calling of Automated Sequencer Traces Using Phred. II. Error probabilities. Genome Res 1998, 8(3): $186-194$.
22. Ewing B, Hillier L, Wendl MC, Green P: Base-Calling of Automated Sequencer Traces Using Phred. I. Accuracy assessment. Genome Res 1998, 8(3): I75-185.

23. Drysdale RA, Crosby MA: FlyBase: genes and gene models. Nucleic Acids Res 2005:D390-395.

24. Wang J, Xia Q, He X, Dai M, Ruan J, Chen J, Yu G, Yuan H, Hu Y, Li R, et al.: SilkDB: a knowledgebase for silkworm biology and genomics. Nucleic Acids Res 2005:D399-402.

25. Altschul SF, Madden TL, Schaffer AA, Zhang J, Zhang Z, Miller W, Lipman DJ: Gapped BLAST and PSI-BLAST: a new generation of protein database search programs. Nucleic Acids Res 1997, 25(I 7):3389-3402

26. Romualdi C, Bortoluzzi S, d'Alessi F, Danieli GA: IDEG6: a web tool for detection of differentially expressed genes in multiple tag sampling experiments. Physiol Genomics 2003, I2(2): 159-162.
Publish with Bio Med Central and every scientist can read your work free of charge

"BioMed Central will be the most significant development for disseminating the results of biomedical research in our lifetime. "

Sir Paul Nurse, Cancer Research UK

Your research papers will be:

- available free of charge to the entire biomedical community

- peer reviewed and published immediately upon acceptance

- cited in PubMed and archived on PubMed Central

- yours - you keep the copyright
BioMedcentral 